\title{
Hypoxia decreases podocyte expression of slit diaphragm proteins
}

Hong Lu'

Gaurav Kapur'

Tej K Mattoo'

William D Lyman ${ }^{1,2}$

'Carman and Ann Adams Department of Pediatrics, ${ }^{2}$ Children's Research

Center of Michigan, Children's

Hospital of Michigan, Detroit, MI, USA

This article was published in the following Dove Press journal: International Journal of Nephrology and Renovascular Disease 17 July 2012

Number of times this article has been viewed

Background: Chronic hypoxia contributes to progressive tubulointerstitial injury and, consequently, renal failure. However, the effect of hypoxia on glomerular podocytes, which are integral to the slit diaphragm complex and responsible for selectivity of the glomerular filtration barrier, has not been completely determined.

Methods: Conditionally immortalized mouse podocyte cells were exposed to hypoxic $\left(1 \% \mathrm{O}_{2}\right)$ or normoxic (room air) conditions for 24,48 , or 72 hours, after which cell viability was determined by MTT assay. Cells were stained with podocin and phalloidin to determine podocin and intracellular actin distribution. Expression of synaptopodin, CD2-associated protein (CD2AP), NcK, transforming growth factor- $\beta 1$ (TGF- $\beta 1$ ), hypoxia-inducible factor (HIF- $1 \alpha$ ) were evaluated by real-time polymerase chain reaction.

Results: Podocytes exposed to hypoxia had significantly reduced viability at $48(87 \%)$ and 72 hours (66\%). There was disarrangement of intracellular filament actin by phalloidin staining, a $30 \%$ weaker fluorescence intensity by podocin staining, significantly reduced expression of synaptopodin (12\%), CD2AP (42\%), NcK (38\%), and increased expression of TGF- $\beta 1$ and P-ERK after hypoxia treatment.

Conclusion: Podocyte exposure to hypoxia leads to reduced viability and SD protein expression, which may explain persistent and/or increasing proteinuria in patients with progressive renal failure. Increased expression of TGF- $\beta 1$ and P-ERK is associated with apoptosis and fibrosis, which could be the link between hypoxia and glomerular injury.

Keywords: podocytes, hypoxia, slit-diaphragm proteins

\section{Introduction}

Podocytes function to maintain the permeability of the glomerular filtration barrier. Studies have established ${ }^{1,2}$ an association between the development of progressive kidney disease and podocyte failure, ${ }^{3-7}$ as seen in gene mutations of podocyte-specific proteins leading to nephrotic syndrome, renal injury, and failure. The slit diaphragm (SD), which is the intercellular junction between the podocyte foot processes, is composed of different proteins synthesized by podocytes such as podocin, nephrin, CD2-associated protein (CD2AP), NcK, ZO-1, and actinin4.$^{8-10}$ The integrity of the $\mathrm{SD}$, as regulated by protein expression in podocytes, plays a key role in regulating cytoskeletal dynamics and signaling in and between the podocytes. ${ }^{10-13}$ This is critical for maintaining the selectivity of the glomerular filtration barrier. Injury to this barrier leads to proteinuria, which is known as one of the important contributors to progressive renal injury. 
Along with proteinuria, current studies have reported chronic hypoxia as one of the most important factors in progressive renal injury. ${ }^{14,15}$ Recent research has viewed hypoxiainduced injury to the tubulointerstitium as the most important factor for progressive renal injury. ${ }^{5,16}$ Despite reports of chronic hypoxia as the common pathogenic mechanism driving the progression of glomerular injury, as in cyanotic congenital heart disease, renal transplant and glomerulosclerosis, ${ }^{17,18}$ the effect of hypoxia on podocytes has yet to be completely elucidated. A recent study evaluating the role of hypoxia-regulated cellular processes has reported increased risk of glomerular disease with von Hippel-Lindau (VHL) gene inactivation. ${ }^{13}$ Studies have also reported the importance of hypoxia-inducible factors (HIF) in glomerular development. ${ }^{19}$

We hypothesize that acute or chronic hypoxia can injure the podocyte and influence SD protein expression. This may have important implications for progression of renal injury, as podocyte injury secondary to hypoxia may be an overlooked but important contributor to progressive, and possibly, irreversible renal injury.

\section{Materials and methods}

This study was conducted using immortalized podocytes, which were kindly given to us by Dr Peter Mundel. ${ }^{20}$ The podocytes were subjected to hypoxic $\left(1 \% \mathrm{O}_{2}, 5 \% \mathrm{CO}_{2}\right.$ balanced with $\mathrm{N}_{2}$ ) and normoxic (room air, 5\% $\mathrm{CO}_{2}$ ) culture conditions per experimental protocol, as detailed below.

\section{Cell culture}

The clonal cell line of conditionally-immortalized mouse podocytes was cultured in RPMI 1640 (Invitrogen, Carlsbad, CA) with $10 \%$ fetal calf serum (Atlanta Biologicals, Lawrenceville, GA), $100 \mathrm{IU} / \mathrm{mL}$ penicillin, and $100 \mu \mathrm{g} / \mathrm{mL}$ streptomycin (MP Biomedicals, Solon, $\mathrm{OH}$ ), along with interferon $\gamma$ (IFN $\gamma, 10 \mathrm{IU} / \mathrm{mL}$ ) (Sigma, St Louis, MO), at $33 \mathrm{C}$ on collagen type $\mathrm{I}(0.1 \mathrm{mg} / \mathrm{mL})$ (Sigma) coated surfaces. The podocytes were differentiated by culturing them in the same medium without IFN $\gamma$ at $37^{\circ} \mathrm{C}$ for $10-12$ days prior to the experiments. Cells from the fifth to the seventeenth passages were used for the experiments. Podocytes were dispensed into a 6 -well plate $\left(3 \times 10^{4}\right.$ cells/well $), 24$-well plate $\left(6 \times 10^{3}\right.$ cells/well $)$ or 96 -well $\left(1.5 \times 10^{3}\right.$ cells/well $)$ plate for differentiation.

Per the experimental protocol, differentiated podocytes were cultured in a hypoxia chamber (Modular Incubator Chamber; Billups-Rothenberg, Del Mar, CA) with gas flow of $1 \% \mathrm{O}_{2}, 5 \% \mathrm{CO}_{2}$ and balance with nitrogen, or in a normoxic condition, (room air, $21 \% \mathrm{O}_{2}, 5 \% \mathrm{CO}_{2}$ ), at $37^{\circ} \mathrm{C}$.

\section{Cell viability}

\section{MTT assay}

MTT assay involves the conversion of the water-soluble MTT [3-(4,5-dimethythiazol-2-yl)-2,5-diphenyltetrazolium bromide] to an insoluble formazan by live cells only. The formazan is then solubilized by sodium dodecyl sulfite (SDS) solution and determination of the optical density at $570 \mathrm{~nm}$ as an estimate of the percentage of live cells. ${ }^{21}$ Upon differentiation of the podocytes, $100 \mu \mathrm{L}$ of culture medium and $10 \mu \mathrm{L}$ of $12 \mathrm{mM}$ MTT (Invitrogen) stock solution were added to each well. Plates were then incubated at $37^{\circ} \mathrm{C}$ for 4 hours, after which $100 \mu \mathrm{L}$ of $10 \%$ SDS- $0.01 \mathrm{M} \mathrm{HCl}$ solution (Sigma) were added and incubated at $37^{\circ} \mathrm{C}$ for $4-18$ hours. The absorbance at $570 \mathrm{~nm}$ was determined using a plate reader (SpectraMax 250; Molecular Devices, Sunnyvale, CA).

\section{Podocin and phalloidin staining}

Cells were differentiated on coverslips for 10-12 days and then fixed for 5 minutes with cold acetone. After being washed three times with Hank's buffered salt solution (Invitrogen), 0.3\% Triton-X 100 was added to the cells. The cells were then treated with $5 \%$ bovine serum albumin for 30 minutes and incubated with rabbit anti-mouse podocin (primary) antibody (1:200; Sigma) and FITC-conjugated goat anti-rabbit (secondary) antibody (Sigma). As controls, cells were stained with rabbit serum and then secondary antibody. Fluorescently conjugated phalloidin (1:40; Molecular Probes Inc, Eugene, OR), which binds to the interface between F-actin subunits, was used for staining the podocytes for evaluation of the cytoskeletal cell framework. The stained cells were then examined under a Nikon Eclipse E-600 fluorescence microscope (480 nm, 20×; Nikon, Tokyo, Japan). The fluorescent intensity was compared using software (Simple PCI, Sewickley, PA).

\section{Real-time PCR}

Total RNA was extracted from cell lysates according to the manufacturer's instructions for the RNeasy Mini kit (Qiagen, Valencia, CA). Briefly, lysis buffer with $\beta$-mercaptoethanol ( $\beta$-ME) was added to the cells, which were then homogenized with syringe, and then the same volume of $80 \%$ ethanol was added. Then, the lysates were applied to a mini-column and washed and digested with DNase I (Qiagen, Valencia, CA), then washed again. RNA samples were analyzed with a Nano-Drop, ND-1000 spectrophotometer (Thermo Scientific, Wilmington, DE); and samples with 260/280 ratio in the range of 1.9-2.2 (indicative of minimal DNA contamination) were selected for further analysis. RNA 6000 Pico Assay and Agilent 2100 Bioanalyzer (Agilent Technologies, Palo Alto, CA) were used 
to determine $18 \mathrm{~S}$ and $28 \mathrm{~S}$ ribosomal bands and ensure no RNA degradation was present. $100 \mathrm{ng}$ of total RNA was reverse transcribed into cDNA using iScript cDNA Synthesis Kit (Bio-Rad, Hercules, CA), and housekeeping genes such as $\beta$-actin and 18S RNA were evaluated as internal controls to normalize the expression of the study genes and comparison between hypoxia and normoxia culture conditions. All real-time PCR primers (Table 1) were synthesized by Sigma-Aldrich (St Louis, MO). After determining the optimal primer concentration, the sample and primers were used, mixed with iQ Sybr Green Supermix for real-time PCR, under an iQ Real-Time thermocycler (BioRad). Each sample was assayed in triplicate for each gene. The thermal cycler conditions were 10 minutes at $95^{\circ} \mathrm{C}$, followed by 40 cycles of 15 seconds at $95^{\circ} \mathrm{C}$, and then 30 seconds at $60^{\circ} \mathrm{C}$, followed by a melting curve.

\section{Western blot}

Cultured cells were scraped of their substrate while on ice with lysis buffer. Cell lysates were then incubated on ice for half an hour and subsequently centrifuged at $9300 \times \mathrm{g}$ for 15 minutes at $4^{\circ} \mathrm{C}$. The supernatant was collected and proteins were measured by BCA protein assay (Pierce, Rockford, IL); $25 \mu \mathrm{g}$ of lysate protein were applied on polyacrylamide gel electrophoresis. The protein was then transferred to the PVC membrane at $90 \mathrm{~V}$ for 2 hours on ice. The PVC membrane was blocked with $10 \%$ skim milk for 1 hour and incubated with anti-phosphorylated ERK (P-ERK) or anti-total ERK (T-ERK) antibodies (1:5000) (Cell Signal Technology, Danvers, MA) for more than 2 hours. After washing (four times), the membrane was incubated with secondary HRP antibody, washed, and developed with an ECL kit (GE Healthcare, Piscataway, NJ).

\section{Results}

\section{Mouse podocytes exhibit decreased viability and proliferation in response to hypoxia}

Podocytes showed decreased viability by MTT assay when cultured in hypoxic conditions for 24 (90\%) hours,
$48(87 \%)$ hours and $72(66 \%)$ hours (Figure 1A). When podocytes were exposed to hypoxia for a fixed period and then cultured in normoxia conditions, the number of cells increased. This could be related to the proliferative cellular response exhibited by the immortalized podocyte cell line under hypoxic culture conditions (Figure 1B).

\section{Hypoxia induces podocyte actin filament disruption}

Phalloidin binds to the filamentous actin (F-actin), allowing the visualization of the podocyte actin framework. Podocytes under normoxia culture conditions displayed a regular arrangement of the podocyte central actin fibers (Figure 2). Cells treated with hypoxia (48 hours) showed disarrangement and disruption of the podocyte actin framework (Figure 2) with phalloidin staining.

Slit diaphragm molecules expression level reduced in podocytes cultured in hypoxia conditions Slit diaphragm molecules, CD2AP, Nck, and synaptopodin (SD-associated protein), were evaluated by real-time PCR, and podocin expression was evaluated by immunofluorescence microscopy. All the molecules showed decreased expression under hypoxia culture conditions. The expression of CD2AP and Nck was significantly reduced $(\mathrm{n}=3$; $P<0.05$ ) (Figure 3 ), while the reduction in synaptopodin was not statistically significant $(P=0.10)$.

\section{Podocin staining under hypoxia} and normoxia condition

Podocin showed weaker staining by immunofluorescence in cells cultured under hypoxic conditions (Figure 4A, left panel) (48-hour treatment) compared to cells cultured under normoxic culture conditions (Figure 4A, right panel). Reduced podocin expression was confirmed by western blot (Figure 4B).

TGF- $\beta 1$ and HIF- $1 \alpha$ expression were studied as mediators of hypoxia-induced podocyte damage (Figure 5).

Table I Primers used for RT-PCR

\begin{tabular}{lll}
\hline Name & Forward sequence & Reverse sequence \\
\hline Synaptopodin & AGAAGCTACAGTTCTGTTCCCGCA & TTCTACAAGAGGCACAAGGCAGGA \\
Beta-actin & TCGTACCACAGGCATTGTGATGGA & TGATGTCACGCACGATTTCCCTCT \\
TGF- $\beta I$ & TACGTCAGACATTCGGGAAGCAGT & AAAGACAGCCACTCAGGCGTATCA \\
HIF-I $\alpha$ & AGCTGAGGACTTTCCAGGTGTTGA & ACACAGGATGCCTTCCTTGGATCT \\
CD2AP & TCAGCCACATCCACAAACCAAAGC \\
NcK & CCAAGCTATTGACCGTGCCATTGA & ATTGTTCAGGGTTCCACTCCACCA \\
I8S RNA & CGCCGCTAGAGGTGAAATTC & AGGTGCCTCTTCGAGACAAGGTTT \\
\hline
\end{tabular}

Abbreviations: TGF- $\beta$ I, transforming growth factor Beta I; HIF-I $\alpha$, Hypoxia-inducible factor I, alpha subunit; CD2AP, CD2-associated protein; NcK, NcK adaptor protein; I8S RNA, I8S ribosomal RNA. 


\section{A}

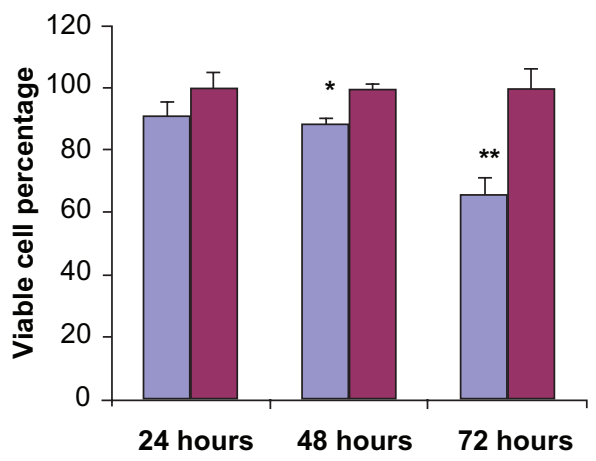

B

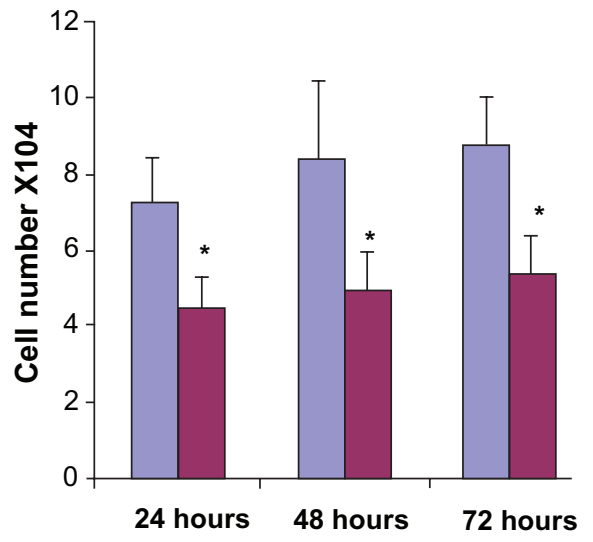

Hypoxia Normoxia

Figure I MTT assay and cell viability of podocytes under hypoxia and normoxia culture conditions. MTT assay (A) showed decreased cell viability under hypoxia treatment at $24(P=0.1 \mathrm{I}), 48$ hours $(* P<0.05)$ and 72 hours $(* * P<0.01)$. (B) Significantly $(P<0.05)$ increased podocyte cell numbers after 24,48 , and 72 hours of hypoxia culture conditions were noticed compared to normoxia culture conditions.

Real-time PCR showed that the relative expression level of TGF- $\beta 1$ increased significantly $(P=0.01)$ after hypoxia treatment, compared to podocytes under normoxia only (expression of $\beta$-actin and 18S RNA was evaluated as internal controls). Activated TGF- $\beta 1$ has a half-life of only a few minutes, and it is primarily degraded within the cell. Therefore, increased expression of TGF- $\beta 1$ in just 30 minutes of hypoxia exposure suggests the role of TGF- $\beta 1$-mediated pathways in podocyte response to hypoxia and subsequent injury.

\section{Phosphorylated ERK expression increased after 48-hour hypoxia treatment}

Protein $(25 \mu \mathrm{g})$ from podocytes exposed to hypoxia and normoxia was isolated to compare total and phosphorylated ERK expression. Compared to normoxia culture conditions,

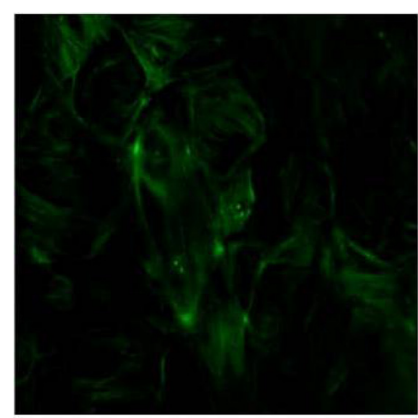

Hypoxia

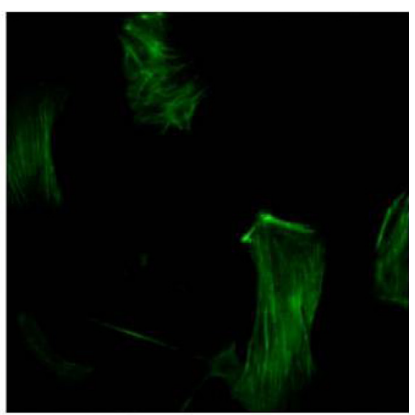

Normoxia
Figure 2 Phalloidin staining to evaluate podocyte actin framework under hypoxia and normoxia culture conditions (20x).

Note: Podocyte under hypoxia conditions showed disarrangement of intracellular actin, compared to the regular arrangement observed under normoxia.
P-ERK expression was increased after 48 hours of hypoxia treatment, while total ERK was unchanged (Figure 6).

\section{Discussion}

Integrity of the glomerular filtration barrier is the single most important factor in preventing proteinuria, a known cause of progressive renal injury. Our study demonstrates that podocytes exposed to hypoxia have decreased expression of some of the several proteins expressed in the SD, along with decreased viability of the podocytes. Several molecules, such as nephrin, podocin, CD2AP, NcK, synaptopodin, ZO-1, actinin4, and P-cadherin, are associated with SD complex. The integrity of the glomerular filtration barrier is dependent on the intimate molecular cross-talk between podocyte foot processes and the $\mathrm{SD} .{ }^{22}$

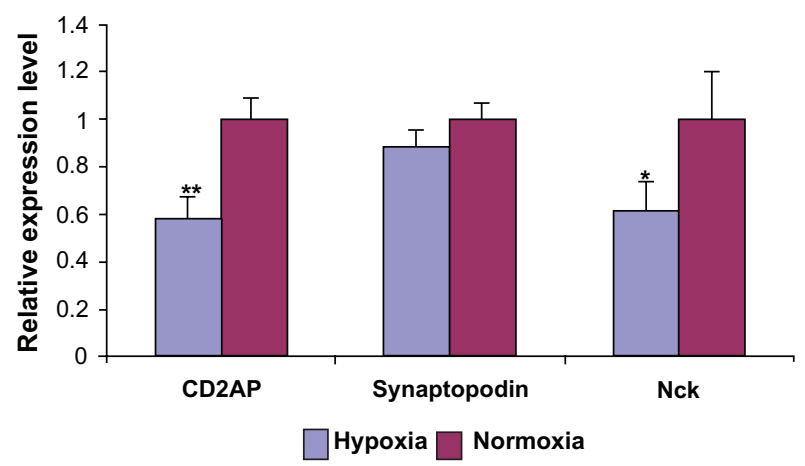

Figure 3 RT-PCR evaluating mRNA expression level of SD molecules CD2AP, synaptopodin, and Nck in podocytes exposed to hypoxia and normoxia.

Note: Reduced podocyte expression of slit diaphragm molecules, CD2AP $(* * P<0.01)$, Nck (*P<0.05), and synaptopodin, was observed after 48 hours of hypoxic culture conditions. 


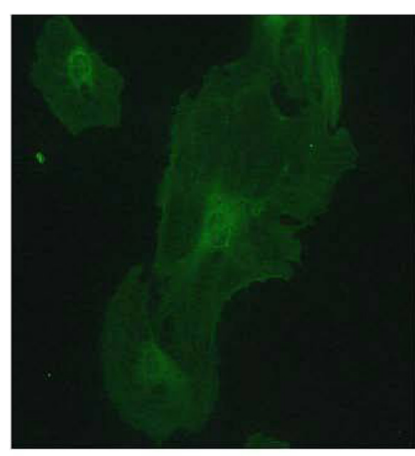

Hypoxia

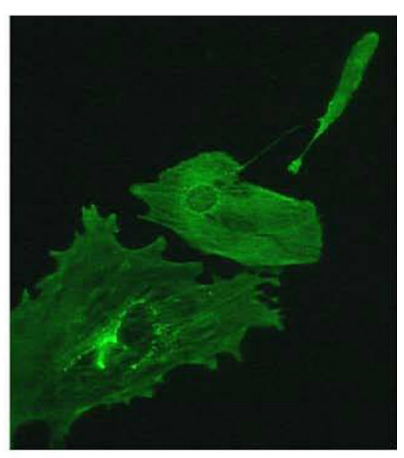

Normoxia
Figure 4A Podocin staining under hypoxia and normoxia culture conditions (20x). Note: Podocytes under hypoxia showed less immunofluorescence when compared to podocytes under normoxia conditions.

Chronic hypoxic-ischemic injury to the renal tubulointerstitium is currently viewed as one of the most important factors in the progression of chronic renal injury. The decreased expression of SD complex proteins and podocyte injury due to hypoxia, as reported herein, could represent an important contributor to progressive renal injury associated with hypoxia. Few studies have focused on the effect of hypoxia on the glomerular podocytes and the slit diaphragm proteins integral to the filtration barrier. A recent study by Brukamp et $\mathrm{al}^{13}$ reported on glomerular injury secondary to alterations in hypoxia-mediated cellular processes involving VHL protein and HIF. In their study model, VHL-deficient mice mimic podocyte hypoxia due to increased activity of HIF (normal cellular response to hypoxia) and subsequent cellular processes stimulated by HIF. The evaluation of podocytespecific genes in VHL-deficient podocyte cell lines showed downregulation of synaptopodin and no change in nephrin and C2DAP expression when evaluating the cells at 0, 24, and 48 hours of hypoxia $\left(\mathrm{O}_{2} 1.5 \%\right)$. Our study shows decreased in vitro expression of synaptopodin, C2DAP, and NcK by RTPCR, as well as decreased staining for podocin and disruption of the podocin cytoskeletal framework in podocytes cultured

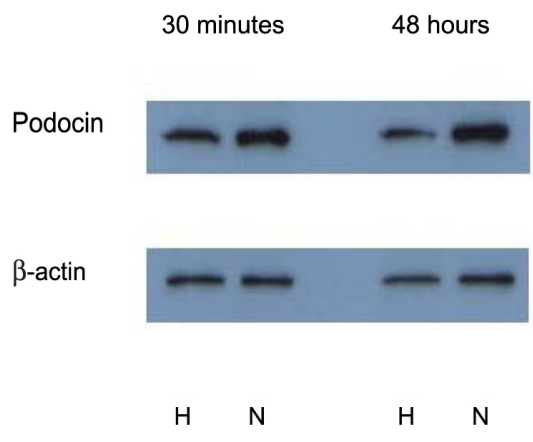

Figure 4B Western blot shows podocin expression reduced under hypoxia treatment, both after 30 minutes and 48 hours. in hypoxia $\left(1 \% \mathrm{O}_{2}\right)$, in comparison to podocytes in normoxic $\left(21 \% \mathrm{O}_{2}\right)$ conditions. It is possible that in VHL inactivation in the study by Brukamp et al, ${ }^{13}$ the podocyte gene expression may have been altered by adenovirus-mediated delivery of Cre recombinase to generate VHL deficient podocyte cell lines. In addition, other studies involving VHL-deficient transgenic mice have revealed different pathophysiologic processes leading to renal failure, such as immune complex mediated renal injury ${ }^{22}$ and crescentric glomerulonephritis, associated with up-regulation of CXCR4. ${ }^{23}$

Proteinuria is a significant contributor to progressive kidney damage; however, the underlying processes, especially hypoxia, have yet to be elucidated in detail. Andersson et $\mathrm{al}^{11}$ reported that mild ischemia (15 minutes, not sufficient to cause tubular injury) reperfusion injury in rats resulted in severe proteinuria and altered the permeability of the glomerular filtration barrier. Electron microscopy of the kidneys did not show any significant changes in the podocytes or glomerular basement membrane. Although ischemia injury followed by reperfusion injury induces the formation of free radicals, the results of our studies provide an alternative injury pathway for the findings reported by Andersson et al. ${ }^{11}$ This is important, as direct injury to the podocytes by hypoxia could initiate or possibly account for persistent injury in acute or chronic renal failure. Our study shows that hypoxia of 30 minutes is associated with increased HIF, which is one of the major factors mediating cellular response to oxygen deprivation. Podocyte injury frequently leads to reorganization of the SD and reorganization of the foot process structure. ${ }^{24}$ Macconi et $\mathrm{al}^{25}$ reported that angiotensin II induces actin cytoskeletal reorganization, thereby altering the permeability of the glomerular filtration barrier. It is currently hypothesized that alterations of the charge in the glomerular basement and integrity of the SD are essential for the integrity of the glomerular filtration barrier. Alteration of the podocyte proteins is expressed in the SD; therefore, the podocyte skeletal framework, as reported herein, is important, as decreased perfusion leading to hypoxia is a common underlying factor in acute or chronic renal injury. Our findings, when viewed in light of other reports of podocyte injury (with or without hypoxia) and the importance of hypoxia in progressive renal injury, point toward an aspect of renal injury (podocyte and hypoxia) which is yet to be elucidated in detail.

It is interesting to note that the immortalized mouse cell line in the study showed a proliferative response to hypoxia. This could be due to the inability of some of the immortalized mouse podocytes (with constitutively active SV40 T antigen) to exit the cell cycle upon differentiation experiments. Podocytes in culture differ from those in vivo, as the initiation of podocyte 
TGF- $\beta$

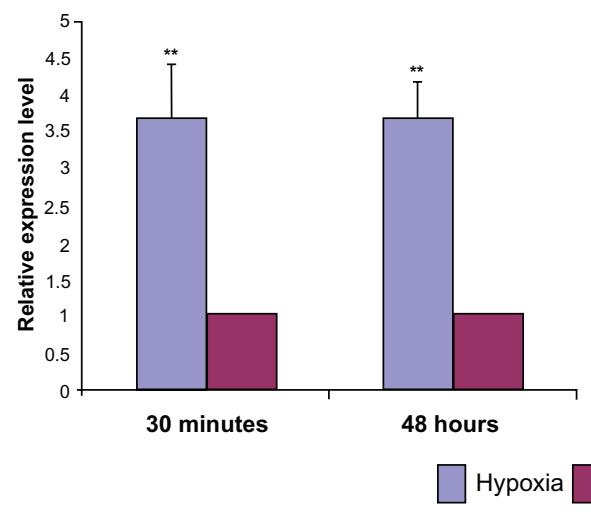

HIF

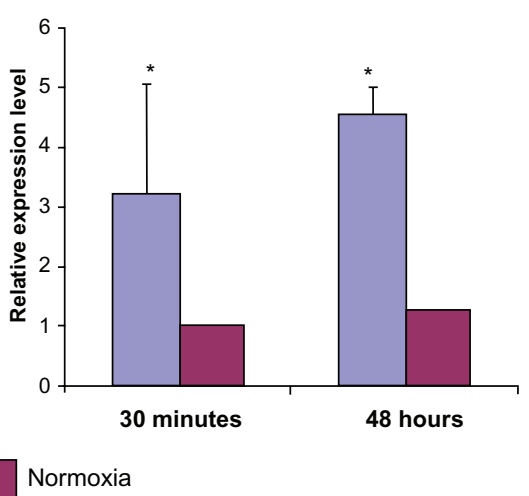

Figure 5 Relative mRNA expression level of TGF- $\beta$ and HIF in podocytes after 30 minutes and 48 hours of hypoxia.

Note: There is significant increase in expression of TGF- $\beta(* * P<0.01)$ and HIF $(* P<0.05)$ after just 30 minutes of podocyte hypoxia exposure. Abbreviations: TGF- $\beta$, transforming growth factor; HIF, hypoxia-inducible factor.

differentiation is intimately linked to the cells leaving the cell cycle and stopping cell division. ${ }^{26}$ Studies have shown that persistent injured podocytes (in vivo experiments) undergo either apoptosis or necrosis, instead of proliferation. ${ }^{27}$

Transforming growth factor- $\beta$ (TGF- $\beta$ ) is an anti-inflammatory and profibrotic cytokine. ${ }^{28}$ The involvement of TGF- $\beta$ in the development of progressive glomerular injury has been reported in cell culture experiments, animal studies, and human disease..$^{29,30,31}$ Woroniecki et al reported that deficiency of CD2AP leads to the development of glomerular lesions resembling human focal segmental glomerulosclerosis, between 3-4 weeks, associated with increased expression levels of TGF- $\beta .^{30}$ There is an early increase in podocyte TGF- $\beta$ expression in nearly all glomerular diseases. ${ }^{31,32}$ Generally, TGF- $\beta$ is considered the important molecule in the cascade of progressive renal fibrosis. ${ }^{33}$ It has numerous effects on the glomerulus, including increased extracellular matrix production, ${ }^{1}$ hypertrophy, and podocyte apoptosis. ${ }^{34}$

The Smad and MAPK pathways mediate the cellular effects of TGF- $\beta 1$. The two pathways interact by certain MAP kinases, such as extracellular signal-regulated protein kinase (ERK), c-Jun N-terminal kinase (JNK), and p38 kinase, ${ }^{35}$ which have been shown to activate or inhibit Smad pathways by phosphorylating Smad proteins. Hayashida et al, ${ }^{35}$ in a study on skin fibroblasts, reported increased expression of fibrogenic connective tissue growth factor secondary to TGF- $\beta$-mediated Smad and ERK pathways. Omori et $\mathrm{al}^{36}$ reported that in rat kidney, the expressions of activated ERK and P-ERK were closely correlated with that of TGF- $\beta$. The increased expressions of TGF- $\beta 1$ and P-ERK in our result suggests early ( 6 hours) and continued (48 hours) activation of fibrogenic pathways in the podocytes subjected to hypoxia.

Our study findings elucidate that hypoxia-induced podocyte injury, as evidenced by decreased cell viability and disruption of glomerular filtration barrier permeability, corresponds with decreased expression of SD podocyte proteins. This could lead to proteinuria, an important mediator of progressive renal injury. This aspect of renal injury needs to be studied in detail, as reversal or slowing of these pathways could affect the outcome of progressive renal injury.

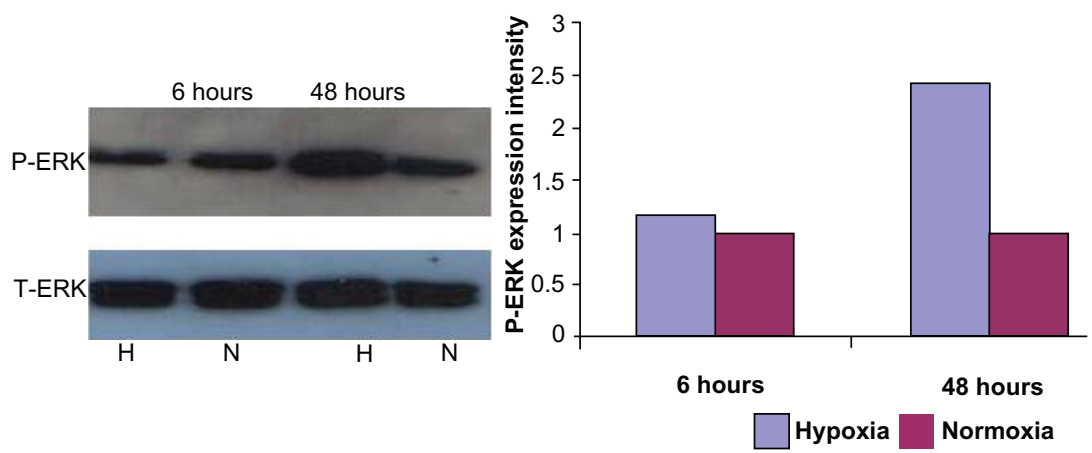

Figure 6 Expression of P-ERK and T-ERK by western blot.

Notes: Podocytes exposed to hypoxic $(\mathrm{H})$ conditions show increased P-ERK expression when compared to podocytes under normoxic (N) conditions. At 48 hours, the increase is almost 2.5 times, suggesting a role of P-ERK-mediated pathways in podocyte response to hypoxia. 


\section{Disclosure}

Originally presented at the annual Pediatric Academic Societies/Society for Pediatric Research Meeting in 2009. No extramural funding was received for this project.

\section{References}

1. Davies DJ, Messina A, Thumwood CM, Ryan GB. Glomerular podocytic injury in protein overload proteinuria. Pathology. 1985;17(3):412-419.

2. Pavenstadt H, Kriz W, Kretzler M. Cell biology of the glomerular podocyte. Physiol Rev. 2003;83(1):253-307.

3. Michaud JL, Kennedy CR. The podocyte in health and disease: insights from the mouse. Clin Sci (Lond). 2007;112(6):325-335.

4. Dittrich S, Kurschat K, Dahnert I, Vogel M, Muller C, Lange PE. Cyanotic nephropathy and use of non-ionic contrast agents during cardiac catherization in patients with cyanotic congenital heart disease. Cardiol Young. 2000;10(1):8-14.

5. Eckardt KU, Bernhardt WM, Weidemann A, et al. Role of hypoxia in the pathogenesis of renal disease. Kidney Int Suppl. 2005;99:S46-S51.

6. Nakatsue T, Koike H, Han GD, et al. Nephrin and podocin dissociate at the onset of proteinuria in experimental membranous nephropathy. Kidney Int. 2005;67(6):2239-2253.

7. Tryggvason K, Pikkarainen T, Patrakka J. Nck links nephrin to actin in kidney podocytes. Cell. 2006;125(2):221-224.

8. Fauchald P, Albrechtsen D, Leivestad T, Pfeffer P, Talseth T, Flatmark A. Renal replacement therapy in patients over 60 years of age. Transplant Proc. 1988;20(3):432-433.

9. Kriz W, Kretzler M, Provoost AP, Shirato I. Stability and leakiness: opposing challenges to the glomerulus. Kidney Int. 1996;49(6):1570-1574.

10. Asanuma K, Mundel P. The role of podocytes in glomerular pathobiology. Clin Exp Nephrol. 2003;7(4):255-259.

11. Andersson M, Nilsson U, Hjalmarsson C, Haraldsson B, Nystrom JS. Mild renal ischemia-reperfusion reduces charge and size selectivity of the glomerular barrier. Am J Physiol Renal Physiol. 2007;292(6): F1802-F1809.

12. Briscoe DM, Kim MS, Lillehei C, Eraklis AJ, Levey RH, Harmon WE. Outcome of renal transplantation in children less than two years of age. Kidney Int. 1992;42(3):657-662.

13. Brukamp K, Jim B, Moeller MJ, Haase VH. Hypoxia and podocytespecific Vhlh deletion confer risk of glomerular disease. Am J Physiol Renal Physiol. 2007;293(4):F1397-F1407.

14. Fine LG, Ong AC, Norman JT. Mechanisms of tubulo-interstitial injury in progressive renal diseases. Eur J Clin Invest. 1993;23(5):259-265.

15. Kaushal GP, Basnakian AG, Shah SV. Apoptotic pathways in ischemic acute renal failure. Kidney Int. 2004;66(2):500-506.

16. Konopka CL, Jurach A, Wender OC. Experimental model for the study of chronic renal ischemia in rats: morphologic, histological and ultrastructural analysis. Acta Cir Bras. 2007;22(1):12-21.

17. Burke TJ, Arnold PE, Schrier RW. Prevention of ischemic acute renal failure with impermeant solutes. Am J Physiol. 1983;244(6):F646-F649.

18. Cutroneo KR. TGF-beta-induced fibrosis and SMAD signaling: oligo decoys as natural therapeutics for inhibition of tissue fibrosis and scarring. Wound Repair Regen. 2007;15 Suppl 1:S54-S60.

19. Bernhardt WM, Schmitt R, Rosenberger C, et al. Expression of hypoxiainducible transcription factors in developing human and rat kidneys. Kidney Int. 2006;69(1):114-122.
20. Mundel P, Reiser J, Zuniga Mejia Borja A, et al. Rearrangements of the cytoskeleton and cell contacts induce process formation during differentiation of conditionally immortalized mouse podocyte cell lines. Exp Cell Res. 1997;236(1):248-258.

21. Kretzler M. Regulation of adhesive interaction between podocytes and glomerular basement membrane. Microsc Res Tech. 2002;57(4): 247-253.

22. Steenhard BM, Isom K, Stroganova L, et al. Deletion of von HippelLindau in glomerular podocytes results in glomerular basement membrane thickening, ectopic subepithelial deposition of collagen \{alpha\} 1 alpha\} 2 alpha\} $1(\mathrm{IV})$, expression of neuroglobin, and proteinuria. Am J Pathol. 2010;177(1):84-96.

23. Ding M, Cui S, Li C, et al. Loss of the tumor suppressor Vhlh leads to upregulation of Cxcr4 and rapidly progressive glomerulonephritis in mice. Nat Med. 2006;12(9):1081-1087.

24. Saleem MA, O’Hare MJ, Reiser J, et al. A conditionally immortalized human podocyte cell line demonstrating nephrin and podocin expression. J Am Soc Nephrol. 2002;13(3):630-638.

25. Macconi D, Ghilardi M, Bonassi ME, et al. Effect of angiotensinconverting enzyme inhibition on glomerular basement membrane permeability and distribution of zonula occludens-1 in MWF rats. J Am Soc Nephrol. 2000;11(3):477-489.

26. Pavenstadt H. Roles of the podocyte in glomerular function. Am J Physiol Renal Physiol. 2000;(2):F173-F179.

27. Sporn MB, Roberts AB. Peptide growth factors are multifunctional. Nature. 1988;332(6161):217-219.

28. Border WA, Okuda S, Languino LR, Sporn MB, Ruoslahti E. Suppression of experimental glomerulonephritis by antiserum against transforming growth factor beta 1. Nature. 1990;346(6282):371-374.

29. Yamamoto T, Nakamura T, Noble NA, Ruoslahti E, Border WA. Expression of transforming growth factor beta is elevated in human and experimental diabetic nephropathy. Proc Natl Acad Sci USA. 1993;90(5): 1814-1818.

30. Woroniecki RP, Schiffer M, Shaw AS, Kaskel FJ, Bottinger EP. Glomerular expression of transforming growth factor-beta (TGF-beta) isoforms in mice lacking CD2-associated protein. Pediatr Nephrol. 2006;21(3):333-338.

31. Schiffer M, Schiffer LE, Gupta A, et al. Inhibitory Smads and TGF- $\beta$ signaling in glomerular cells. J Am Soc Nephrol. 2002;13(11): 2657-2666.

32. Bottinger EP. TGF-beta in renal injury and disease. Semin Nephrol. 2007;27(3):309-320.

33. Schnaper HW, Hayashida T, Hubchak SC, Poncelet AC. TGF-beta signal transduction and mesangial cell fibrogenesis. Am J Physiol Renal Physiol. 2003;284(2):F243-F252.

34. Lim IJ, Phan TT, Tan EK, et al. Synchronous activation of ERK and phosphatidylinositol 3-kinase pathways is required for collagen and extracellular matrix production in keloids. J Biol Chem. 2003;278(42): 40851-40858.

35. Hayashida T, Decaestecker M, Schnaper HW. Cross-talk between ERK MAP kinase and Smad signaling pathways enhances TGF-beta-dependent responses in human mesangial cells. FASEB J. 2003;17(11):e1576-e1578.

36. Omori S, Kitagawa H, Koike J, et al. Activated extracellular signalregulated kinase correlates with cyst formation and transforming growth factor-beta expression in fetal obstructive uropathy. Kidney Int. 2008;73(9):1031-1037.

\section{Publish your work in this journal}

The International Journal of Nephrology and Renovascular Disease is an international, peer-reviewed open-access journal focusing on the pathophysiology of the kidney and vascular supply. Epidemiology, screening, diagnosis, and treatment interventions are covered as well as basic science, biochemical and immunological studies. The journal welcomes
Dovepress

original research, clinical studies, reviews \& evaluations, expert opinion and commentary, case reports and extended reports. The manuscript management system is completely online and includes a very quick and fair peerreview system, which is all easy to use. Visit http://www.dovepress.com/ testimonials.php to read real quotes from published authors. 